EXTENDED REPORT

\title{
Arthrographic joint distension with saline and steroid improves function and reduces pain in patients with painful stiff shoulder: results of a randomised, double blind, placebo controlled trial
}

\author{
R Buchbinder, S Green, A Forbes, S Hall, G Lawler
}

Ann Rheum Dis 2004;63:302-309. doi: 10.1136/ard.2002.004655

See end of article for authors' affiliations

....................

Correspondence to: Associate Professor R Buchbinder, Department of Clinical Epidemiology,

Suite 41 Cabrini Medical Centre, 183 Wattletree Rd, Malvern, Victoria,

Australia 3144;

rachelle.buchbinder@

med.monash.edu.au

Accepted 12 June 2003

\begin{abstract}
Objective: To determine whether arthrographic distension with a mixture of saline and steroid, in patients with painful stiff shoulder for at least 3 months, is better than placebo in improving function, pain, and range of motion at 3,6 , and 12 weeks.

Methods: A randomised, placebo controlled trial with participant and outcome assessor blinding in which shoulder joint distension with normal saline and corticosteroid was compared with placebo (arthrogram). Outcome measures, assessed at 3, 6, and 12 weeks, included a shoulder-specific disability measure (SPADI), a patient preference measure (Problem Elicitation Technique (PET)), pain, and range of active motion.

Results: From 96 potential participants, 48 were recruited. Four withdrew from the placebo group after the 3 week assessment and three subsequently received arthrographic distension with saline and steroid. At 3 weeks, significantly greater improvement in SPADI $(p=0.005)$, PET, overall pain, active total shoulder abduction, and hand behind back was found in participants in the joint distension and steroid group than in the placebo group. At 6 weeks the results of the intention to treat analysis favoured joint distension, although the between-group differences were only significant for improvement in PET (difference in mean change in PET between groups $=45.9$ (95\% Cl 3.2 to 88.7). Excluding the four withdrawals, the betweengroup differences for the disability and pain measures significantly favoured distension over placebo. At 12 weeks, both the intention to treat analysis and an analysis excluding the four withdrawals demonstrated a significantly greater improvement in PET score for the distension group.

Conclusions: Short term efficacy of arthrographic distension with normal saline and corticosteroid over placebo was demonstrated in patients with painful stiff shoulder.
\end{abstract}

$\mathrm{P}$ ainful stiff shoulder (also termed frozen shoulder or adhesive capsulitis) is a common cause of shoulder pain, estimated to affect $2-5 \%$ of the general population. ${ }^{12}$ It is characterised by spontaneous onset of pain and progressive stiffness of the glenohumeral joint accompanied by significant disability. ${ }^{3}$ Although early studies suggested a self limiting condition lasting for an average of $2-3$ years, ${ }^{5}$ later studies have found that up to $40 \%$ of patients have persistent symptoms and restricted movement beyond 3 years, ${ }^{6}$ and $15 \%$ have persistent disability. ${ }^{7}$ After systematically reviewing the evidence, we previously concluded that there were insufficient data to either support or refute the efficacy of any of the studied interventions for this condition and further well designed clinical trials were needed. ${ }^{8}$

Arthrographic distension of the glenohumeral joint capsule leading to capsular rupture was first described as a potential treatment for painful stiff shoulder by Andren and Lundberg in 1965. ${ }^{9}$ They described the injection of $20 \mathrm{ml}$ contrast medium (and normal saline if a larger amount of fluid was required) into the joint, which was then allowed to flow back into the syringe. This was repeated several times or until capsular rupture. Since then there have been descriptions of distension of the joint being achieved with various substances, including combinations of saline, local anaesthetic, steroid, contrast, and air. ${ }^{10-15}$ Most have included corticosteroid as part of the procedure..$^{10-13} 15$ Six case series and one non-randomised controlled trial of arthrographic distension (mostly with corticosteroid) have reported promising results $^{10-17}$; however, there have been no placebo controlled trials.

Three published randomised controlled trials have compared distension with or without steroid with corticosteroid alone. ${ }^{18-20}$ Two trials failed to show any benefit for distension and corticosteroid over corticosteroid injection alone, ${ }^{18}{ }^{19}$ while the third trial reported a significant improvement in range of motion and analgesic use in the group treated with distension and steroid compared with steroid alone, but no difference in pain. ${ }^{20}$ These trials injected small volumes (9$10 \mathrm{ml}$ or up to $20 \mathrm{ml}$, respectively), which may have been insufficient to achieve the desired effect and may not have included end points capable of detecting clinically important change. ${ }^{8}$ In addition, although intra-articular corticosteroid injection alone is commonly used to treat the painful stiff shoulder, there is currently limited evidence to either support or refute its use. ${ }^{21}$

A recent Cochrane systematic review identified only one placebo controlled trial of intra-articular corticosteroid injection for painful stiff shoulder. ${ }^{21}{ }^{22}$ This double blind trial reported no differences in pain and range of movement up to 6 months between intra-articular steroid injection and placebo. ${ }^{22}$ Another trial, in which neither the participants nor outcome assessment was blinded, compared intraarticular corticosteroid injection with no treatment (analgesia alone) and reported a significant benefit of injection up to 6 weeks. ${ }^{23}$ A further trial of 19 participants compared a combination of both intra-articular and subacromial steroid 


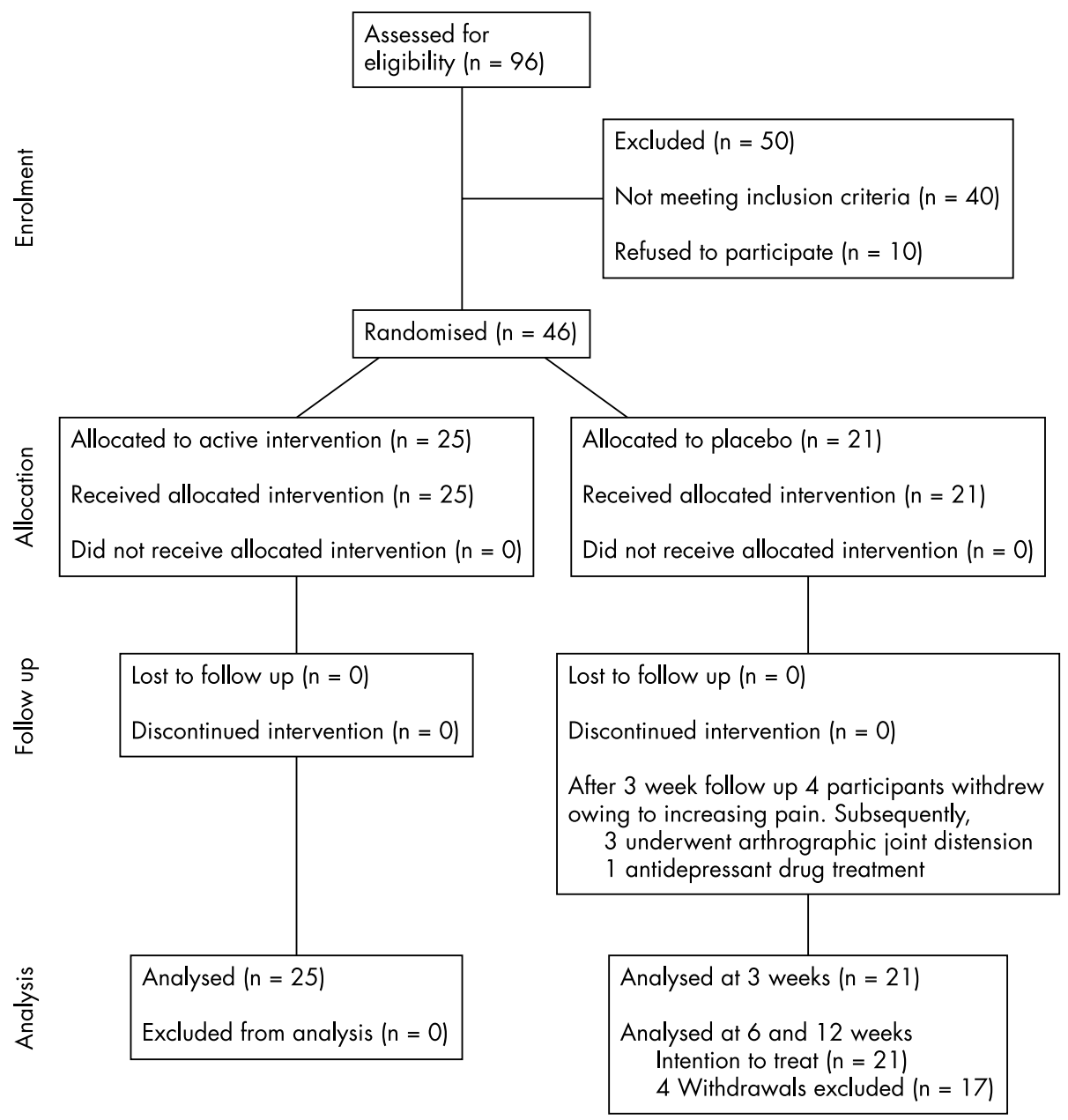

Figure 1 Movement of participants through the trial.

injection with no treatment and reported little difference in the long term outcome but some early benefit of injection in reduced pain and increased range of movement. ${ }^{24} \mathrm{~A}$ fourth trial compared $10 \mathrm{mg}$ and $40 \mathrm{mg}$ triamcinolone and found a non-significant trend towards improvement in pain at 6 weeks with the higher dose intra-articular steroid injection, but no differences between the effect of the doses on sleep disturbance, functional impairment, or improvement in external rotation. ${ }^{25} \mathrm{~A}$ second placebo controlled trial of intra-articular corticosteroid for adhesive capsulitis of the shoulder, including 93 participants, has been reported since publication of the Cochrane review. ${ }^{26}$ Carette et al compared four interventions: fluoroscopically guided intra-articular steroid or saline injection either alone or followed by supervised physiotherapy. All participants were taught a home exercise programme. They reported a significant improvement in pain and disability at both 6 weeks and 3 months in the treatment groups who received intraarticular steroid, with added physiotherapy providing faster improvement in shoulder range of motion. With time, differences between the treatment groups who received corticosteroid and those that did not became smaller and by 12 months all treatment groups had improved to a similar degree in all the measured outcomes. ${ }^{26}$

To determine whether arthrographic distension of the shoulder joint with normal saline and corticosteroid as described in the literature is of benefit for the painful stiff shoulder, we performed a randomised, double blind, controlled trial. In the absence of any well established proven treatments for this condition we used placebo as the comparator. Our study aimed at determining whether arthrographic distension with normal saline and corticosteroid, in patients with painful stiff shoulder of at least 3 months, is better than placebo in improving function, pain, and range of motion at 3 weeks, and secondly, at determining whether benefit is maintained at 6 and 12 weeks.

\section{MATERIALS AND METHODS}

Study design and treatment assignment

We performed a randomised, double blind, placebo controlled trial. Consenting patients were randomised by computer generated, simple randomisation in permuted blocks of six to receive either arthrographic distension or sham distension (arthrogram) (meaning that there were three arthrographic distensions and three placebo allocations in random order within each block of six patients). ${ }^{27}$ Allocation concealment was ensured and the study biostatistician kept the assignment scheme. A master list of intervention by enrolment number was supplied to the radiologist performing the technique who was not involved in any other part of the trial, and there was no access possible by the outcome assessor, enrolling physicians, or study participants. The Cabrini Hospital ethics committee granted ethical approval.

\section{Study participants}

We recruited study participants from the general practice referral base of the community based rheumatology practices of two of the investigators ( $\mathrm{RB}, \mathrm{SH})$. Inclusion criteria were 
pain and stiffness in predominantly one shoulder for $\geqslant 3$ months; restriction of passive motion of greater than $30^{\circ}$ in two or more planes of movement, measured to onset of pain with a gravity inclinometer; and adults $>18$ years of age. Exclusion criteria were severe pain at rest, defined as $>7$ out of 10 on a visual analogue scale; previous arthrographic distension; systemic inflammatory joint disease (including rheumatoid arthritis, polymyalgia rheumatica); radiological evidence of osteoarthritis of the shoulder or fracture; calcification about the shoulder joint; reason to suspect a complete rotator cuff tear (weakness of arm elevation, a positive "drop arm sign", a high riding humerus visible on $x$ ray examination of the shoulder, or demonstration of a complete rotator cuff tear on ultrasound); contraindications to arthrogram and/or distension such as current warfarin treatment; allergy to local anaesthetic or iodinated contrast; pregnancy; likely not to comply with follow up (for example, living too far away to attend for follow up assessment and/or those indicating they would be unable and/or unwilling to attend for outcome assessment); or lack of written informed consent.

\section{Interventions}

One radiologist (GL) performed all interventions according to a standardised protocol. Care was taken to ensure that all participants in both groups were handled in an identical manner. Participants were positioned in a supine position with the affected arm externally rotated and a sandbag on the hand. The image intensifier was centred on the glenohumeral joint, cones open to include scapula and upper third of the humerus, and the image intensifier to tabletop distance set at $50 \mathrm{~cm}$. The skin was marked for arthrogram needle site and infiltrated with local anaesthetic. The arthrogram needle was positioned, connected to connector tap and tube, and $0.5-1 \mathrm{ml}$ of contrast injected to confirm the needle position. A further $6 \mathrm{ml}$ of contrast was injected and an $x$ ray picture taken. This concluded the procedure for the placebo group.

For the active group, $40 \mathrm{mg}$ Depo-Medrol (methylprednisolone acetate; $1 \mathrm{ml}$ ) and up to $82 \mathrm{ml}$ normal saline was then injected (total volume $30-90 \mathrm{ml}$ ). One of the following constituted the end point of the procedure: filling of the subscapular bursa; capsular rupture; injection of the total $90 \mathrm{ml}$ volume; participant requesting termination of the procedure. The procedure was also terminated if there was severe pain, an allergic reaction to the injected materials, or if a complete rotator cuff tear was diagnosed.

Participants in both groups received a simple exercise programme comprising pendular exercises and scapular setting (isometric scapular retraction). Participants were asked to stop taking any non-steroidal anti-inflammatory drugs, but were allowed paracetamol and codeine preparations. No manual treatments (for example, physiotherapy, massage, chiropractic) or other medical interventions (for example, intra-articular steroid injection) were allowed during the study.

\section{Outcome assessment}

The same "blinded" outcome assessor (a manipulative physiotherapist, SG) evaluated all patients at baseline, and 3, 6, and 12 weeks after the intervention. Data collected at baseline included personal details and clinical characteristics, including duration of symptoms, severity of the condition, and previous treatment. If not already available, an $x$ ray picture of the shoulder was obtained to ensure the participant met the selection criteria.

\section{Disability assessment}

The Shoulder Pain and Disability Index (SPADI) is a self administered, shoulder-specific, fixed-item index consisting of 13 items divided into two subscales: pain (five items) and disability (eight items). ${ }^{28}$ Responses to each item were recorded on a 10 point Likert scale, where $0=$ no pain or no difficulty and $9=$ worst imaginable pain or so difficult it required help, for the pain and disability items, respectively. The SPADI score is calculated by summing then averaging the items of the two subscales to give a score out of 100 (higher score indicates more pain/disability). It has acceptable testretest reliability (intraclass correlation coefficients $=0.91$ and 0.65 (95\% confidence interval (CI) 0.42 to 0.8 ) in surgical and primary care groups, respectively $)^{29}$ and responsiveness. ${ }^{30}$

The Problem Elicitation Technique (PET) is a patient preference disability measure administered by an interviewer. $^{31}$ In contrast with conventional questionnaires, patient preference questionnaires ask the patient to identify their own problems related to the disease that they would most like to see improve as a result of treatment. The importance and magnitude of each identified problem is elicited by Likert scales, where $0=$ not at all important to $10=$ most important, and $0=$ without any difficulty to $7=$ unable to do, respectively. Each problem is scored by multiplying the magnitude of the problem (level of difficulty, frequency, or degree of severity) by the importance of the problem. A higher score indicates a higher degree of perceived disability and/or importance. A PET score was derived by summing the scores for all volunteered problems.

\section{Pain perception}

Participant's overall assessment of pain was measured using a vertical Likert scale, labelled "no pain" at the bottom (0) and "maximum imaginable pain" at the top (10).

\section{Range of active motion}

A standardised protocol was used to measure active total shoulder flexion and abduction (that is, scapular and

\begin{tabular}{|c|c|c|}
\hline Variable & $\begin{array}{l}\text { Distension with } \\
\text { saline and } \\
\text { steroid }(n=25) \\
\text { Mean (SD) }\end{array}$ & $\begin{array}{l}\text { Placebo } \\
\text { ( } n=21 \text { ) } \\
\text { Mean (SD) }\end{array}$ \\
\hline Age (years) & $57.2(8.6)$ & $57.5(8.1)$ \\
\hline $\begin{array}{l}\text { Duration of symptoms (days), median } \\
\text { (range) }\end{array}$ & $118(102-194)$ & $\begin{array}{l}114(96- \\
402)\end{array}$ \\
\hline Severity (out of 10) & $6.5(2.4)$ & $7.0(2.0)$ \\
\hline $\begin{array}{l}\text { Shoulder Pain and Disability Index } \\
\text { Score }\end{array}$ & $53.8(18.5)$ & $55.7(22.6)$ \\
\hline Problem Elicitation Technique Score & $172.6(48.2)$ & $169.9(76.7)$ \\
\hline $\begin{array}{l}\text { Overall pain score } \\
\text { Range of motion (degrees) }\end{array}$ & $6.0(2.0)$ & $5.7(2.1)$ \\
\hline Total shoulder flexion & $108.6(24.5)$ & $98.3(22.7)$ \\
\hline Total shoulder abduction & $79.8(27.5)$ & $72.9(21.2)$ \\
\hline \multirow{3}{*}{$\begin{array}{l}\text { External rotation in neutral } \\
\text { Hand behind back }\end{array}$} & $41.7(20.2)$ & $35.8(27.0)$ \\
\hline & $16.0(3.9)$ & $16.7(4.2)$ \\
\hline & No (\%) & No $(\%)$ \\
\hline Female & $20(80)$ & $17(74)$ \\
\hline \multicolumn{3}{|l|}{ Level of education completed } \\
\hline Primary & $6(30)$ & $4(21)$ \\
\hline Secondary & $6(30)$ & $12(63)$ \\
\hline Tertiary & $8(40)$ & $3(16)$ \\
\hline Diabetes & 8 (32) & $5(24)$ \\
\hline Previous corticosteroid injection & 7 (28) & $6(28.6)$ \\
\hline Post operative capsulitis & $0(0)$ & $1(4.8)$ \\
\hline \multicolumn{3}{|l|}{ Occupation } \\
\hline Manual & $3(12)$ & $0(0)$ \\
\hline Non-manual & $14(56)$ & $12(57)$ \\
\hline Retired/unemployed & $8(32)$ & $9(43)$ \\
\hline Dominant arm affected & $17(68)$ & $9(43)$ \\
\hline
\end{tabular}




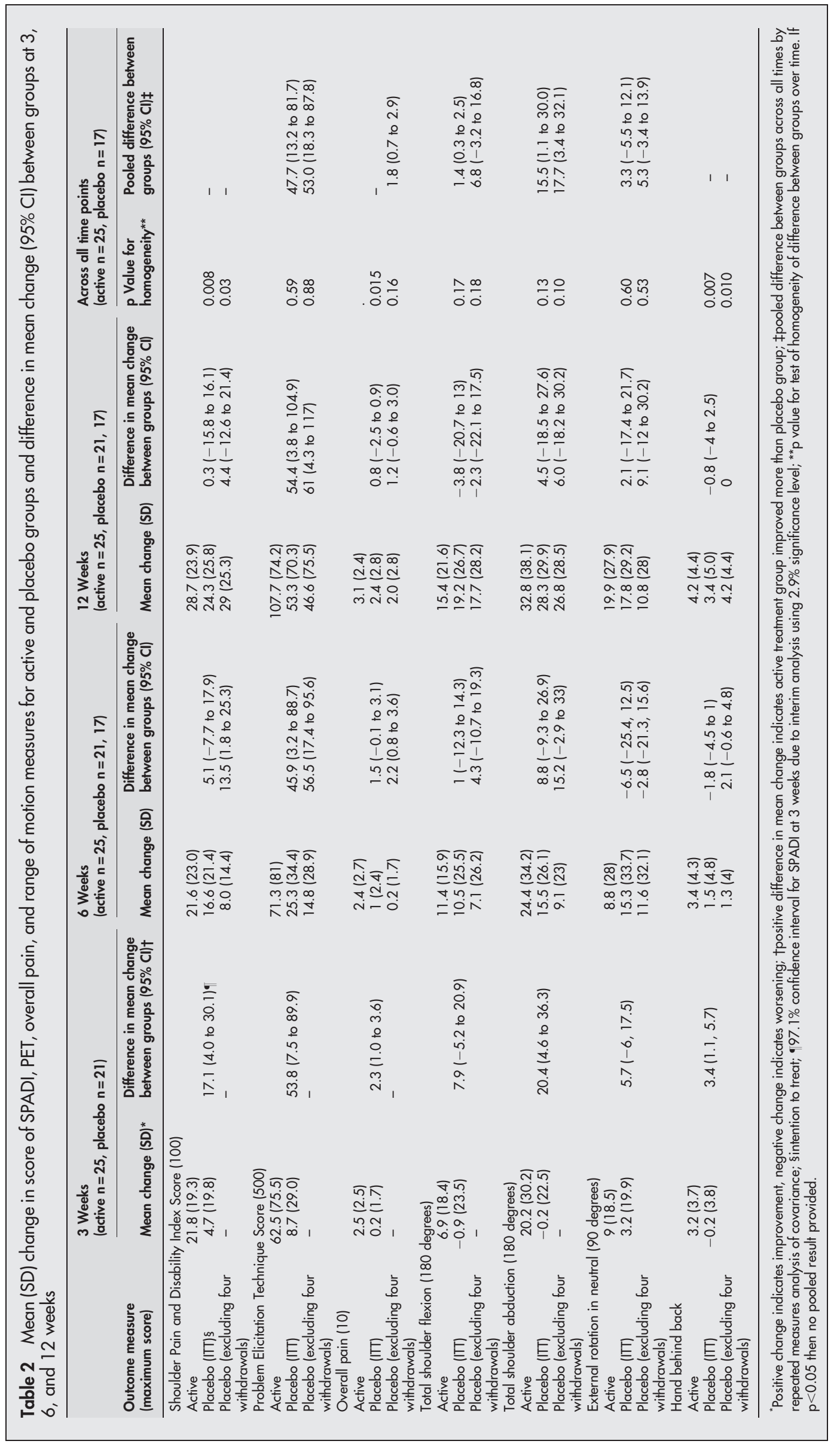


glenohumeral movement) and external rotation in neutral using an inclinometer, and hand behind back was measured by assessment of the anatomical landmark reached by the extended thumb. ${ }^{32}$ When performed by manipulative therapists this method has been shown to have high intrarater reliability (intraclass correlation coefficients $0.75-0.93$ ). ${ }^{32}$

\section{Adverse effects}

Adverse effects of the intervention were elicited by the use of open ended questions. Information about the number of analgesics taken each day were also collected.

\section{Sample size and power}

The primary outcome measure, determined before the start of the trial was the SPADI score at 3 weeks. A previous study found that differences in the SPADI score of $>10$ indicate clinically important improvement (or worsening) of shoulder function. ${ }^{28}$ However, the standard deviation of this difference was not reported. We determined that a sample size of 45 participants in each group had $90 \%$ power to detect a difference in mean SPADI scores of $\geqslant 10$ if the standard deviation was $\leqslant 15$. The target sample size was increased to 50 in each group to allow for a possible $10 \%$ withdrawal.

\section{Statistical methods}

All analyses was carried out using SPSS ${ }^{33}$ and Stata software (Statacorp, College Station, Texas, USA). Demographic and prognostic factors at baseline were compared. An intention to treat analysis was performed. Mean and standard deviation of change from baseline was calculated at all time points for all outcome measures. Differences in mean change between the distension and control groups were calculated, and their significance assessed by an independent samples $t$ test, establishing p values and 95\% confidence intervals.

Because recruitment was slow an unplanned interim analysis was made. We chose a liberal stopping rule, which allocates equal significance levels $(p<0.029)$ to both interim and final analyses of the SPADI scores at the 3 week follow up while maintaining an overall 5\% significance level across the two equally spaced analyses. ${ }^{34}$ Analyses of secondary end points were performed at conventional 5\% significance levels. As these analyses demonstrated a significant effect of distension for the SPADI, as well as the PET and overall pain at 3 weeks, recruitment in the trial was terminated. At the time of the interim analysis, all randomised patients had completed all follow up visits.

There were no withdrawals from the trial in the distension group, but four patients in the placebo group withdrew after the 3 week assessment because of persisting pain and disability. After unblinding of treatment assignment, three of the four subsequently underwent arthrographic joint distension with saline and corticosteroid, although all four patients continued to attend for follow up and the outcome assessor remained blinded. Because an intention to treat analysis at 6 and 12 weeks is likely to be an underestimation of the effect of distension, an alternative analysis omitting these four subjects was also completed. Other intermediate
Table 4 Adverse effects associated with distension with saline and steroid and placebo. Results are shown as No (\% of total number in both groups)

\begin{tabular}{|c|c|c|}
\hline Adverse effect & $\begin{array}{l}\text { Distension with } \\
\text { saline and steroid } \\
(\mathrm{n}=25 \text { ) }\end{array}$ & $\begin{array}{l}\text { Placebo } \\
(n=21)\end{array}$ \\
\hline Pain associated with procedure & $4(16)$ & $1(4)$ \\
\hline $\begin{array}{l}\text { Increased pain for up to } 48 \text { hours } \\
\text { after procedure }\end{array}$ & $3(12)$ & $1(4)$ \\
\hline Claustrophobia at time of procedure & $1(4)$ & $1(4)$ \\
\hline Unsettled, anxious and hot & $0(0)$ & $1(4)$ \\
\hline Shoulder noisy (that is, fluid noises) & $1(4)$ & $0(0)$ \\
\hline Total reported adverse effects & $9(36)$ & $4(19)$ \\
\hline
\end{tabular}

analyses, in which values for the 6 and 12 week assessments for these four subjects were estimated from overall patterns among study completers in the placebo group, were also performed (results not presented here but are available from the authors).

To determine whether differences between treatment groups were constant over time, we performed two repeated measures assessments for non-homogeneity: a global (two degree of freedom) test for any variation in group differences across the 3, 6, and 12 week time points; and a (one degree of freedom) test for systematically increasing or decreasing group differences over time. As the latter test is more sensitive than the global test for such systematic effects, the $p$ value for this linear trend in group differences over time was reported. If no evidence of such differences in treatment effect across time was found with either test (at a 5\% significance level), a pooled difference between groups across all time points was calculated.

\section{RESULTS}

Ninety six potential participants were screened and 48 recruited. Reasons for exclusion were a condition other than painful stiff shoulder (rotator cuff disease, osteoarthritis, pain referred from the cervical spine) $(n=30)$, pain greater than 7 out of $10(n=5)$, pain for less than 3 months $(n=1)$, taking warfarin $(\mathrm{n}=4)$, and refusal of informed consent $(\mathrm{n}=8)$. Two recruited participants withdrew before the intervention, leaving a total sample of 46 (48\% of those screened). Figure 1 shows the way in which participants moved through the trial.

No important differences were found between the active and placebo groups for any of the demographic or clinical variables (table 1). The median (range) volumes injected into the distension and placebo groups were $43.3 \mathrm{ml}(2 \mathrm{l}-80)$ and $6 \mathrm{ml}(6-6)$, respectively.

Table 2 presents the mean change from baseline for disability, pain, and range of motion measures for both groups for all times, and the between-group difference of that change. The results of both the intention to treat analysis and the alternative analysis omitting the four withdrawals are presented at 6 and 12 weeks. At 3 weeks, there was a

Table 3 Results for the four participants in the placebo group who withdrew from the trial after the 3 week assessment

\begin{tabular}{|c|c|c|c|c|c|c|c|c|c|c|c|c|}
\hline & \multicolumn{4}{|c|}{ SPADI (week) } & \multicolumn{4}{|c|}{ PET (week) } & \multicolumn{4}{|c|}{ Overall pain (week) } \\
\hline & 0 & 3 & 6 & 12 & 0 & 3 & 6 & 12 & 0 & 3 & 6 & 12 \\
\hline Participant 1 & 76 & 71 & 14 & 22 & 240 & 210 & 120 & 90 & 4 & 3 & 1 & 2 \\
\hline Participant 2 & 63 & 77 & 2 & 2 & 50 & 60 & 20 & 20 & $8^{*}$ & 9 & 1 & 1 \\
\hline Participant 3 & 45 & 34 & 19 & 19 & 110 & 150 & 100 & 60 & 5 & 6 & 3 & 3 \\
\hline Participant 4 & 46 & 29 & 3 & 0 & 140 & 140 & 118 & 70 & 5 & 5 & 0 & 0 \\
\hline
\end{tabular}


significantly greater improvement in SPADI (at the required $2.9 \%$ significance level), PET, overall pain, active total shoulder abduction, and hand behind back in participants for the distension group compared with the placebo group. There was a trend favouring distension also observed for active total shoulder flexion and external rotation in neutral.

At 6 weeks, the intention to treat analysis favoured distension but was only significant for improvement in PET (difference in mean change in PET between groups $=45.9$ (95\% CI 3.2 to 88.7). When the four withdrawals were excluded from the analysis, the between-group differences for the disability and pain measures significantly favoured distension over placebo (difference in mean change in SPADI between groups $=13.5$ (95\% CI 1.8 to 25.3 ) and difference in mean change in overall pain between groups $=2.2(95 \%$ CI 0.8 to 3.6 ).

At 12 weeks, there was a significantly greater improvement in the PET score for the distension group by both intention to treat analysis (difference in mean change in PET between groups $=54.4(95 \%$ CI 3.8 to 104.9$)$ and when the four withdrawals were excluded from the analysis (difference in mean change in PET between groups $=61 \quad(95 \%$ CI 4.3 to 117).

Difference between treatment groups appeared constant over time $(p>0.05)$ for all outcomes except SPADI, overall pain (intention to treat analysis only), and hand behind back (table 2). Although there also seemed to be a tendency for difference between treatment groups to decrease over time for active total shoulder flexion and abduction, these were not statistically significant. The results of the pooled difference between groups across all times for those measures where the linear trend test failed to achieve statistical significance are shown in the last column of table 2. This confirms an overall benefit of distension over placebo over 12 weeks as measured by PET, overall pain (analysis excluding withdrawals), and total shoulder abduction.

Table 3 presents the raw data at each time for the four participants who withdrew from the trial after the 3 week assessment. Three of the withdrawals received distension subsequent to the 3 week assessment, and all four participants had improved substantially according to all assessed measures by the 6 week assessment; this was largely sustained at 12 weeks.

No serious adverse effects were reported by participants in either group, but more participants in the active group had pain at the time of the procedure or lasting up to 48 hours than in the placebo group (table 4).

There was no significant difference in the number of analgesics taken each day between the active and placebo group at any time during the study, although there was a trend for subjects in the placebo group to consume more analgesia at 3 weeks compared with baseline (mean (SD) number of analgesics a day at baseline and 3 weeks in the active group and placebo groups was 0.75 (1.75) and 0.64 (1.5), and 1.11 (2.02) and 1.47 (3.47), respectively; difference in mean change at 3 weeks -0.45 (95\% CI -1.31 to 1.41$)$ ).

\section{DISCUSSION}

We have shown that arthrographic distension with normal saline and corticosteroid in patients with painful stiff shoulder of at least 3 months is better than placebo in improving function, pain, and range of movement at 3 weeks. Furthermore, this benefit appeared to be maintained at 6 weeks, although the sustained gain over placebo at 12 weeks was only observed for function when measured by a patient preference questionnaire.

Four participants withdrew from the placebo group after the week 3 assessment and three subsequently underwent distension. We performed and presented the 6 and 12 week analysis with these participants both included (intention to treat analysis), and excluded from the analysis. We have also provided their raw data (table 3). The condition of these patients clearly improved after distension therapy, but we cannot attribute this improvement directly to the distension. The intention to treat analysis attributes this improvement to the placebo group, and hence probably underestimates the true effect of distension. We regard the intention to treat analysis as being conservative, in that treatment group crossovers only occurred in one direction (that is, placebo to distension for four participants after the 3 week assessment), and these patients responded very quickly thereafter. The intention to treat analysis at 6 and 12 weeks, therefore, represents distension versus placebo and distension dilution. The analysis excluding these four participants corresponds broadly to substitution of the mean placebo responses at each time point for these four participants, with a corresponding penalty of four degrees of freedom in the calculation of standard deviations and $t$ statistics. More formal methods with various assumptions of mechanisms of missing data are possible but are not presented here.

This is the first reported trial that has compared arthrographic distension with saline and corticosteroid with placebo. The propensity for painful stiff shoulder to gradually resolve over time makes it imperative that any study of efficacy of a new treatment includes either a comparator with a known effect, or placebo in the absence of interventions of proved efficacy. Classic adhesive capsulitis evolves through three phases-an early painful phase, an intermediate stiff phase during which the stiffness predominates and the pain is less pronounced, and a final recovery phase characterised by gradual return of movement. ${ }^{35}$ We postulated, a priori, that distension may be most effective in the intermediate phase and therefore only included patients who had had at least 3 months of symptoms and whose resting pain was less than 7 out of 10. In the early painful phase patients may be unable to tolerate distension of the capsule. By the 12 week assessment we observed substantial improvement in the placebo control group, in keeping with the natural history of this condition. We also formally tested for systematically increasing or decreasing group differences over time. There was a statistically significant reduction in treatment group differences over time for SPADI, overall pain (using the intention to treat), and hand behind back, with a nonsignificant trend for decreasing group differences also seen for active total shoulder flexion and abduction. This is also consistent with a favourable natural history.

Possibly, the observed improvements in the distension group were partially or entirely due to the injection of corticosteroid rather than capsular distension. We think this is unlikely in view of the fact that over $25 \%$ of participants received one or more steroid injections before their entry into the trial without benefit. On the other hand, we cannot discount the possibility that this lack of benefit might have arisen as a result of incorrect needle placement. There are currently no published placebo controlled studies comparing "blinded" injection with radiologically guided intra-articular steroid injection for painful stiff shoulder.

Two previous negative trials of distension and steroids used intra-articular steroid injection (without distension) as the comparison group. ${ }^{18}{ }^{19}$ Jacobs et al included 47 participants and compared distension using $6 \mathrm{ml}$ local anaesthesia and $3 \mathrm{ml}$ air (total volume $9 \mathrm{ml}$ ) alone with intra-articular steroid injection alone with combined distension and intra-articular steroid (total volume $10 \mathrm{ml}$ ). ${ }^{19}$ Participants were offered a total of three injections at 6 week intervals unless they either refused or had a dramatically improved range of movement and no pain, and the final assessment was performed at 16 weeks. Just as in our study, all patients reported 
improvement during the study. Although the mean improvements in abduction and forward flexion appeared significantly greater in the steroid with distension and steroid only groups compared with the distension only group, these results were not statistically significant. Data for improvement in pain were not presented according to treatment group. Corbeil et al included 45 participants and compared arthrogram and distension using local anaesthetic and corticosteroid at a volume of $20 \mathrm{ml}$ or capsular rupture, with arthrogram and intra-articular steroid injection alone. ${ }^{18}$ More than $80 \%$ of patients in both treatment groups improved, and there were no significant differences in either pain or limitation of movement at 1 and 3 months between the groups. ${ }^{18}$ A third trial of 22 participants also compared arthrographic distension using local anaesthetic and corticosteroid at a volume of $20 \mathrm{ml}$ with intra-articular steroid injection alone. ${ }^{20}$ The treatment was repeated once a week for a maximum of 6 weeks or until no symptoms. Although the results up to 12 weeks for flexion, external rotation and extension (but nor abduction), analgesic use, and physicians' impression of severity of symptoms (but not pain at rest or with function) are reported to significantly favour the distension group, only graphical data are presented.

Several explanations are possible for the observed lack of additional benefit of distension with steroid over intraarticular steroid alone. These include insufficient distension due to the low volumes injected, type 2 error due to the small number of patients studied in both negative trials, and the lack of sensitive end points. Although all three trials used some measure of pain and range of motion to determine response to treatment, none included a measure of function.

Corticosteroid injections are also commonly used to treat other painful shoulder conditions, although the evidence for their efficacy in these disorders is also limited. ${ }^{21}$ For example, for rotator cuff disease, we identified seven trials that compared subacromial steroid injection with placebo for rotator cuff disease $\mathrm{e}^{36-42}$; one trial that compared supraspinatus tendon injection with placebo $^{43}$; and one trial that compared intra-articular steroid injection with placebo. ${ }^{44}$ Only the results of two trials involving a total of 45 participants could be pooled. ${ }^{36}{ }^{39}$ There was a small benefit of subacromial steroid injection over placebo at four weeks for pain (SMD 0.83, 95\% CI 0.39 to 1.26 ), function (SMD 0.63 (95\% CI 0.20 to 1.06 ), and range of active abduction (SMD $0.82,95 \%$ CI 0.39 to 1.25$).{ }^{21}$ Of the remaining unpooled trials, two reported some benefit of injection over placebo, ${ }^{37}{ }^{40}$ two reported no differences between the treatment groups, ${ }^{38} 42$ and one trial reported greater improvement in pain at 3 and 12 months in the placebo group. ${ }^{41}$ No difference in pain or consumption of analgesics at 2 and 8 weeks' follow up was reported in the single trial that compared supraspinatus tendon injection with placebo. ${ }^{43}$ No benefit of steroid injection over placebo was demonstrated at 4 weeks in pain, range of abduction, or success of treatment in the single trial that examined the efficacy of intra-articular steroid. ${ }^{44}$

We chose to use the SPADI, a shoulder-specific pain and disability index, as the primary end point in this trial in view of the importance of these end points to the patient. Empirical studies have demonstrated that it fulfils criteria for an evaluative instrument. One possible explanation for its failure to demonstrate significant benefit of distension over placebo at 12 weeks is a ceiling effect or inability to detect improvements beyond the measurement capabilities of the index. The disability items in the SPADI consist of basic activities of daily living, such as washing hair and dressing, but do not include more complex daily activities, such as sporting endeavour and recreational pursuits.
We therefore also included a patient preference questionnaire on the grounds that some clinically important benefits of new treatments, if present, may not be detected in individual patients by fixed item questionnaires. ${ }^{45}$ For example, it has been shown that many problems identified as important by the patient with rheumatoid arthritis do not appear in conventional questionnaires, and even the most commonly identified disabilities are either not mentioned at all or classified as unimportant by many other patients. ${ }^{45}$ By tailoring of the items to the specific disabilities of the individual patient, it is suggested that "noise" created by including items not relevant to the specific patient will be reduced, thereby enhancing the responsiveness to detect change. Unlike the SPADI, no ceiling effect was observed for the PET, which showed significant improvement in the distension group compared with placebo at 12 weeks even when the withdrawals were included in an intention to treat analysis. Further exploratory work is planned comparing the SPADI and PET instruments.

Range of abduction improved significantly at 3 weeks, but significant differences between groups were not sustained. The failure to demonstrate benefit of distension on glenohumeral joint mobility at 3 months may be attributable to a type 2 error. The original sample size calculation was performed on the primary end point (the SPADI) and hence this study may have been underpowered to assess differences in range of motion. Alternatively, the beneficial effects of distension demonstrated by this trial may not be related to improvement in range of motion. Previous work has demonstrated a lack of correlation between range of motion and disability, and hence it is possible that the improvement in disability was attributable to the demonstrated improvement in pain.

Because recruitment in this trial was slower than expected, we elected to perform an unplanned interim analysis when about half of the patients had completed follow up. We controlled the overall $5 \%$ significance level by selecting a liberal significance level (0.029) for the interim analysis and accepted the possible penalty of requiring a reduced significance level (also 0.029) for the final analysis. ${ }^{34}$ The results in table 2 indicate that we would have reached the same decision to end the trial even if a more conservative plan for early interim analyses had been selected (for example, $p=0.005$ for the O'Brien-Fleming plan). ${ }^{34}$

We encountered difficulties with slow patient recruitment. Difficulties in recruiting patients with adhesive capsulitis resulting in early trial termination have been reported previously. ${ }^{26}$ We recruited patients from the general practice referral base of the community based rheumatology practices of two investigators, and we believe that our sample is representative of patients encountered in primary care. Further, the baseline demographic and clinical characteristics outlined in table 1 are similar to those of previous studies. ${ }^{26}$ To enhance recruitment we offered to see patients within one week of referral and we sent out frequent reminders of our study. Thirty of $96(31 \%)$ patients assessed for eligibility had shoulder pain attributable to causes other than painful stiff shoulder, suggesting that general practitioners are poor at recognising this clinical entity and/or they were taking advantage of the short waiting time for their patients to be seen. We believe that other methods to enhance recruitment, such as advertising for volunteers would have increased the proportion of ineligible patients screened in our setting.

Arthrographic distension of the shoulder with steroid and saline is already widely accepted, available, and affordable. This is probably the most important factor responsible for slow patient recruitment, with participants and referrers reluctant to accept the $50 \%$ chance of placebo when there is ready access to this treatment. Our trial highlights the 
difficulties of performing placebo controlled trials in conditions where the active intervention is already widely accepted. It also raises the more fundamental concern of making new treatments available as part of standard care before their proper evaluation.

In summary, we have shown that shoulder joint distension with a combination of saline and steroid for patients with a painful stiff shoulder of at least 3 months significantly improves function, pain, and range of movement at 3 weeks, and this benefit appears to be maintained at 6 weeks. There were no appreciable adverse effects. Further research is required to determine whether shoulder joint distension with a combination of corticosteroid and saline is better than either distension with saline alone or corticosteroid injection alone; whether repeat distensions with or without steroid extend the benefit; and whether physiotherapy targeted to mobilisation and exercise enhance the benefit of joint distension with steroid.

\section{ACKNOWLEDGEMENT}

This work was supported by a research grant from the Cabrini Clinical Education and Research Institute.

\section{Authors' affiliations}

R Buchbinder, Department of Clinical Epidemiology, Cabrini Hospital and Department of Epidemiology and Preventive, Medicine, Monash University, Victoria, Australia

S Green, A Forbes, Department of Epidemiology and Preventive Medicine, Monash University, Victoria, Australia

S Hall, Department of Medicine, Monash University and Cabrini Medical Centre, Victoria, Australia

G Lawler, St Francis Xavier Cabrini Hospital, Victoria, Australia

\section{REFERENCES}

1 Anton HA. Frozen shoulder. Can Fam Phys 1993;39:1773-77.

2 Lundberg B. The frozen shoulder. Acta Orthop Scand 1969;119(suppl):5-59.

3 Croft P, Rigby AS, Boswell R, Schollum J, Selman A. The prevalence of chronic widespread pain in the general population. J Rheumatol 1993;20:710-13.

4 Neviaser TJ. Adhesive capsulitis. Orthop Clin North Am 1987;18:439-43.

5 Reeves B. The natural history of the frozen shoulder syndrome. Scand J Rheumatol 1975;4:193-6.

6 Simmonds FA. Shoulder pain; with particular reference to the "frozen" shoulder. J Bone Joint Surg Br 1949;31:426-32.

7 Hazleman BL. The painful stiff shoulder. Rheumatol Phys Med 1972;11:413-21.

8 Green S, Buchbinder R, Glazier R, Forbes A. Systematic review of randomised controlled trials of interventions for painful shoulder: selection criteria outcome assessment, and efficacy. BMJ 1998:316:354-60.

9 Andren L, Lundberg BJ. Treatment of rigid shoulders by joint distension during arthrography. Acta Orthop Scand 1965;36:45-53.

10 Ekelund A, Rydell N. Combination treatment for adhesive capsulitis of the shoulder. Clin Orthop Relat Res 1992;282:105-9.

11 Fareed DO, Gallivan WR Jr. Office management of frozen shoulder syndrome: treatment with hydraulic distension under local anesthesia. Clin Orthop Relat Res 1989;242:177-83.

12 Gavant M, Rizk T, Gold R, Flick P. Distension arthrography in the treatment of adhesive capsulitis of the shoulder. J Vasc Intervent Radiol 1994:5:305-8.

13 Loyd JA, Loyd HM. Adhesive capsulitis of the shoulder: arthrographic diagnosis and treatment. South Med J 1983;76:879-83.

14 Older MWJ. Distension arthrography of the shoulder joint. In: Bayley I, Kessel L, eds. Shoulder surgery. Berlin: Springer, 1982:123-7.

15 Mulcahy KA, Baxter AD, Oni OOA, Finlay D. The value of shoulder distension arthrography with intraarticular injection of steroid and local anaesthetic: a follow-up study. Br J Rheumatol 1994;67:263-6.

16 Royen B, Pavlov P. Treatment of frozen shoulder by distension and manipulation under local anaesthesia. Int Orthop 1996;20:207-10.

17 Hsu SYC, Chan KM. Arthroscopic distension in the management of frozen shoulder. Int Orthop 1991;15:79-83.

18 Corbeil V, Dussault RG, Leduc BE, Fleury J. Capsulite rétractile de l'épaule: étude comparative de l'arthrographie avec corticothérapie intra-articulaire avec ou sans distension capsulaire. Can Assoc Radiol J 1992;43:127-30.
19 Jacobs LGH, Barton MAJ, Wallace WA, Ferrousis J, Dunn NA, Bossingham DH. Intra-articular distension and steroids in the management of capsulitis of the shoulder. BMJ 1991;302:1498-501.

20 Gam AN, Schydlowsky P, Rossel I, Remvig L, Jensen EM. Treatment of "frozen shoulder" with distension and glucocorticoid compared with glucocorticoid alone. Scand I Rheumatol 1998;27:425-30

21 Buchbinder R, Green S, Youd J. Corticosteroid injections for shoulder pain. (Cochrane review). In: The Cochrane Library, Issue 1. Oxford: Update Software, 2003.

22 Rizk T, Pinals R, Talaiver A. Corticosteroid injections in adhesive capsulitis: investigation of their value and site. Arch Phys Med 1991;72:20-2.

23 Lee $M$. Haq $A$, Wright $V$, Longton $E$. Periarthritis of the shoulder: a controlled trial of physiotherapy. Physiotherapy 1973;59:312-15.

24 Bulgen D, Binder A, Hazleman B, Dutton J, Roberts S. Frozen shoulder: prospective clinical study with an evaluation of three treatment regimes. Ann Rheum Dis 1984;43:353-60.

25 De Jong BA, Dahmen R, Hogeweg JA, Marti RK. Intra-articular triamcinolone acetonide injection in patients with capsulitis of the shoulder: a comparative study of two dose regimens. Clin Rehabil 1998;12:211-15.

26 Carefte S, Moffet H, Tardif J, Bessette L, Morin F, Fremont P, et al. Intraarticular corticosteroids, supervised physiotherapy, or a combination of the two in the treatment of adhesive capsulitis of the shoulder. Arthritis Rheum 2003;48:829-38.

27 Altman DG, Dore CJ. Randomisation and baseline comparison in clinical trials. Lancet 1990;335:149-53

28 Williams J, Holleman D, Simel D. Measuring shoulder function with the shoulder pain and disability index. J Rheumatol 1995;22:727-32.

29 Roach KE, Budiman-Mak E, Songsiridej N, Lertratanakul Y. Development of a shoulder pain and disability index. Arthritis Care Res 1991;4:143-9.

30 Heald S, Riddle D, Lamb R. The shoulder pain and disability index: the construct validity and responsiveness of a region-specific disability measure. Phys Ther 1997;77:1079-89.

31 Buchbinder R, Bombardier C, Yeung M, Tugwell P. Which outcome measures should we use in rheumatoid arthritis clinical trials: responsiveness to treatment of clinical and quality of life measures in a randomized controlled trial. Arthritis Rheum 1995; 38:1568-80.

32 Green S, Buchbinder R, Forbes A, Bellamy N. A standardized protocol for measurement of range of movement of the shoulder using the plurimeter- $V$ inclinometer and assessment of its intrarater and interrater reliability. Arthritis Care Res 1998;11:43-52.

33 SPSS. SPSS for Windows. Release 9.0.1 (24 Feb 1999). In: Standard, ed. 1989-99.

34 Geller N, Pocock S. Interim analyses in randomised clinical trials: ramifications and guidelines for practitioners. Biometrics 1987; 43:213-23.

35 Neviaser RJ, Neviaser TJ. The frozen shoulder: diagnosis and management. Clin Orthop Relat Res 1987;223:59-64.

36 Adebajo AO, Nash P, Hazleman BL. A prospective double blind dummy placebo controlled study comparing triamcinolone hexacetonide injection with oral diclofenac $50 \mathrm{mg}$ TDS in patients with rotator cuff tendinitis. J Rheumatol 1990; 17:1207-10.

37 Blair B, Rokito AS, Cuomo F, Jarolem K, Zuckerman JD. Efficacy of injections of corticosteroids for subacromial impingement syndrome. J Bone Joint Surg Am 1996;78:1685-9.

38 Kirkley A, Litchfield R, Alvarez C, Herbert S, Griffin S. Prospective double blind randomized clinical trial of subacromial iniection of betamethasone and xylocaine versus xylocaine alone in rotator cuff tendinosis [abstract]. J Bone Joint Surg $B r$ 1999;81(suppl 1):107.

39 Petri M, Dobrow R, Neiman R, Whiting-O'Keefe Q, Seaman WE. Randomized, double-blind, placebo-controlled study of the treatment of the painful shoulder. Arthritis Rheum 1987;30:1040-5.

40 Plafki C, Steffen R, Willburger RE, Wittenberg RH. Local anaesthetic injection with and without corticosteroids for subacromial impingement syndrome. Int Orthop 2000;24:40-2.

41 Strobel G. Long-term therapeutic effect of different intra-articular injection treatments of the painful shoulder-effect on pain, mobility and work capacity. Rehabilitation 1996;35:176-8.

42 Vecchio PC, Hazleman BL, King RH. A double-blind trial comparing subacromial methylprednisolone and lignocaine in acute rotator cuff tendinitis. Br J Rheumatol 1993;32:743-5.

43 Withrington RH, Girgis FL, Seifert MH. A placebo-controlled trial of steroid injections in the treatment of supraspinatus tendonitis. Scand J Rheumatol 1985;14:76-8.

44 Berry H, Fernandes L, Bloom B, Clark RJ, Hamilton EB. Clinical study comparing acupuncture, physiotherapy, injection and oral anti-inflammatory therapy in shoulder-cuff lesions. Curr Med Res Opin 1980;7:121-6.

45 Tugwell P, Bombardier C, Buchanan WW, Goldsmith CH, Grace E, Hanna B. The MACTAR Patient Preference Disability Questionnaire-an individualized functional priority approach for assessing improvement in physical disability in clinical trials in rheumatoid arthritis. J Rheumatol 1987;14:446-51. 